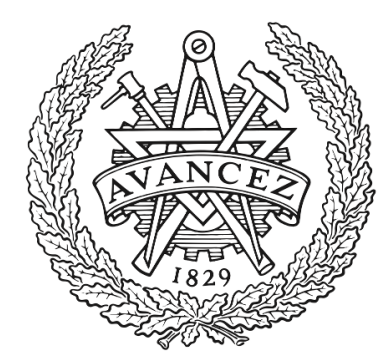

\title{
CHALMERS
}

UNIVERSITY OF TECHNOLOGY

\section{Joint CKF-PHD Filter and Map Fusion for 5G Multi-cell SLAM}

Downloaded from: https://research.chalmers.se, 2023-04-26 12:15 UTC

Citation for the original published paper (version of record):

Kim, H., Granström, K., Gao, L. et al (2020). Joint CKF-PHD Filter and Map Fusion for 5G

Multi-cell SLAM. IEEE International Conference on Communications, 2020-June.

http://dx.doi.org/10.1109/ICC40277.2020.9149211

N.B. When citing this work, cite the original published paper. 


\title{
Joint CKF-PHD Filter and Map Fusion for 5G Multi-cell SLAM
}

\author{
Hyowon Kim*, Karl Granström ${ }^{\dagger}$, Lin Gao $^{\ddagger}$, Giorgio Battistelli ${ }^{\ddagger}$, Sunwoo Kim*, and Henk Wymeersch ${ }^{\dagger}$ \\ * Department of Electronics and Computer Engineering, Hanyang University, Seoul, Korea \\ $\dagger$ Department of Electrical Engineering, Chalmers University of Technology, Sweden \\ † Dipartimento di Ingegneria dell’Informazione, Università degli Studi di Firenze, Italy
}

\begin{abstract}
G is expected to enable simultaneous vehicle localization and environment mapping (SLAM). Furthermore, vehicular networks will be covered with 5G small cells, wherein the map information is collected at each base station (BS) and then fused so as to promote the overall performance of SLAM. In $5 \mathrm{G}$ multicell SLAM, there are challenges such as the unknown number of targets, uncertainty regarding the association between the targets and the measurements, unknown types of targets, as well as map management among BSs. To address those challenges, we propose a new method for 5G multi-cell SLAM which comprises a joint cubature Kalman filter and multi-model probability hypothesis density, and a map fusion routine. Simulation results demonstrate that the proposed method solves the aforementioned challenges and also improves vehicle state and map estimates.

Index Terms-5G multi-cell SLAM, joint CKF, map fusion, message passing, PHD
\end{abstract}

\section{INTRODUCTION}

The ability to collect time and angular measurements, in combination with high resolvability of multipath enables precise positioning in $5 \mathrm{G} \mathrm{mmWave} \mathrm{cellular} \mathrm{networks} \mathrm{[1].} \mathrm{In}$ 5G simultaneous localization and mapping (SLAM) [2]-[5], the main purpose is to estimate the user/vehicle state (location, heading, and clock bias) and build maps of the propagation environment, in which the main objects that reflect or scatter the 5G signal are referred to as targets. 5G SLAM has several challenges [6]: i) target misdetections due to the imperfect detection at the receiver; ii) false alarms generated by channel estimation error or passing targets; and iii) association ambiguities on target-originated measurements and target typeoriginated measurements.

There have been several contributions dealing with these challenges of 5G SLAM [2]-[8], based on a number of methodologies: geometry, message passing (MP) [9], [10], or random finite set (RFS) theory [11], [12]. The geometric relations of the reflected signal are used in [7], [8], but the unknown number of targets and data association (DA) uncertainty are not considered. MP-based 5G SLAM were proposed in [2]-[5]. In [3], [4], it is assumed that there is perfect clock synchronization between the user and base station (BS). In [2]-[4] only large objects are considered, not small scattering points (SPs). Lack of synchronization and different target types was considered in [5], but the uncertainty of DA was evaluated outside the MP process.

An alternate approach to 5G SLAM is to apply the RFS formalism, as in [6]. In general, RFS-based SLAM [11], [13],
[14] is an approach for probabilistic modelling the target and measurement sets under unknown DA, and avoiding a priori determination of the number of targets. It can be combined with multiple target models, as shown in [15], [16]. While different RFS-SLAM approaches are possible, the probability hypothesis density (PHD) filter [17] is computationally very efficient, thanks to the fact that it, via approximation, avoids an explicit enumeration of data association hypotheses. Nevertheless, for non-linear problems such as the 5G PHD-SLAM filter, even when implemented with a computationally efficient RaoBlackwellized particle filter (RBPF), has a complexity that scales exponentially in the vehicle dimension.

In multi-cell scenarios, there are additional challenges, including map management, fusion between different vehicles and between different BSs. Map fusion has been extensively studied [18]-[22]. The generalized covariance intersection (GCI) method [18]-[20], which computes the intersection of information, and is a suitable method for scenarios with a high clutter rate. On the other hand, the arithmetic average (AA) method [21], [22] takes the union of the local information, and is robust to a high missed detection rate.

In this paper, we propose a new method for $5 \mathrm{G}$ multi-cell SLAM by combining MP and RFS formalisms, in order to benefit from the low computational complexity of the former and the flexibility of the latter. In order to cope with different fields-of-view (FoV), we adopt the AA fusion rule. The map fusion routine is to unify local environments. We apply a PHD map representation as in [6], while messages generated by MP in the local filter are computed using a joint cubature Kalman filter (CKF) approach [23].

\section{MODELS AND PROBLEM FORMULATION}

\section{A. Vehicle and Environment Model}

We consider a $5 \mathrm{G}$ multi-cell scenario, with a $\mathrm{BS}$ in the center of each small cell. The BSs are linked to a control center (CC) via backhaul, and the vehicles and BSs are equipped with array antennas. Let us denote ${ }^{1}$ the state of vehicle $n$ at time $k$ by $\mathbf{s}_{k}^{(n)}=\left[\mathbf{v}_{k}^{(n)}, \alpha_{k}^{(n)}, \xi_{k}^{(n)}, \zeta_{k}^{(n)} B_{k}^{(n)}\right]^{\top}$, which consists of the vehicle location $\mathbf{v}_{k}^{(n)}=\left[x_{\mathrm{v}, k}^{(n)}, y_{\mathrm{v}, k}^{(n)}, z_{\mathrm{v}, k}^{(n)}\right]^{\top}$, heading $\alpha_{k}^{(n)}$, translation speed $\xi_{k}^{(n)}$, turn-rate $\zeta_{k}^{(n)}$, and clock bias $B_{k}^{(n)}$. We consider the vehicle movement, which follows the known

\footnotetext{
${ }^{1}$ We will use the following indexing: vehicle $n$, BS $a$, target type $m$, cubature point $c, e, g$, Gaussian mixture component $j$, and time step $k$.
} 
dynamic model with vehicle transition density $f\left(\mathbf{s}_{k}^{(n)} \mid \mathbf{s}_{k-1}^{(n)}\right)$ of the form:

$$
\mathbf{s}_{k}^{(n)}=\mathrm{v}\left(\mathbf{s}_{k-1}^{(n)}\right)+\mathbf{q}_{k},
$$

where $v(\cdot)$ is a vehicle transition function (see [24, Chapter 5]), and $\mathbf{q}_{k}$ denotes zero-mean Gaussian process noise with known covariance $\mathbf{Q}_{k} \in \mathbb{S}_{7}^{+}$, where $\mathbb{S}_{d}^{+}$denotes a $d \times d$ positive semi-definite matrix.

The cell size is $r_{\mathrm{SC}}$, while the maximum transmission range is denoted by $r_{\max }>r_{\mathrm{SC}}$. The transmitted signal from the BS is reflected by surfaces such as walls which characterize virtual anchors (VAs) and scattering points (SPs) such as small objects; the VAs and SPs are defined as targets [5], [6]. We denote by $\mathbf{x}_{k}=\left[x_{k}, y_{k}, z_{k}\right]^{\top}$ the location of a target, and by $m \in\{\mathrm{BS}, \mathrm{VA}, \mathrm{SP}\}$ the target type; multiple targets are modelled by the RFS $\mathcal{X}_{k}$ with the set density $f\left(\mathcal{X}_{k}\right)$ [12], [17]. The BS locations are known, and all target locations are fixed. Thus, the target movement and appearance of new targets are modeled by the RFS-target transition density $f\left(\mathcal{X}_{k} \mid \mathcal{X}_{k-1}, \mathbf{s}_{k}^{(n)}\right)[11]$.

\section{B. Observation Model}

When vehicle $n$ receives signals from BS $a$ the following channel parameters for signal path $l$ are observed by the channel estimation process at the receiver [25]: time-of-arrival (TOA) $\tau_{k, l}^{(a, n)}$, azimuth and elevation for direction-of-arrival (DOA) $\boldsymbol{\theta}_{k, l}^{(a, n)}$ and direction-of-departure (DOD) $\phi_{k, l}^{(a, n)}$. We denote the measurement RFS at time $k$ by $\mathcal{Z}_{k}$; it has elements

$$
\mathbf{z}_{k, l}^{(a, n)}=\mathrm{h}\left(\mathbf{s}_{k}^{(n)}, \mathbf{x}_{k}, m\right)+\mathbf{r}_{k}
$$

where $\mathrm{h}\left(\mathbf{s}_{k}^{(n)}, \mathbf{x}_{k}, m\right)=\left[\tau_{k, l}^{(a, n)}, \boldsymbol{\theta}_{k, l}^{(a, n) \top}, \boldsymbol{\phi}_{k, l}^{(a, n) \top}\right]^{\top}$ is the known measurement function, ${ }^{2}$ and $\mathbf{r}_{k}$ denotes zero-mean Gaussian measurement noise with known covariance $\mathbf{R}_{k} \in$ $\mathbb{S}_{5}^{+}$. Furthermore, we consider missed detections and false alarms. ${ }^{3}$ A vehicle can receive signals from more than 1 BS and these signals are assumed to be orthogonal.

\section{Problem Formulation}

Given the vehicle prior $f\left(\mathbf{s}_{0}^{(n)}\right)$, the purpose of the proposed method is to estimate the vehicle state and to map the propagation environment.

\section{SLAM WITH RFS AND MP}

In this section, we will address posterior density given the measurements for local SLAM in each small cell through representing the marginalized density as the message and belief by the MP rules [9], [10]. Thus, we drop the vehicle index $n$.

\footnotetext{
${ }^{2}$ For target type $m \in\{\mathrm{BS}, \mathrm{VA}, \mathrm{SP}\}$, each element is calculated by geometric relations, details of which are described in [2], [5], [6].

${ }^{3}$ Target detection probability is denoted $p_{\mathrm{D}, k}\left(\mathbf{s}_{k}^{(n)}, \mathbf{x}_{k}, m\right) \in[0,1]$. False alarms are caused by channel estimation errors, and false measurements $\mathbf{z}_{k, l}^{(a, n)}$ are modelled by the clutter intensity $c(\mathbf{z})$.
}

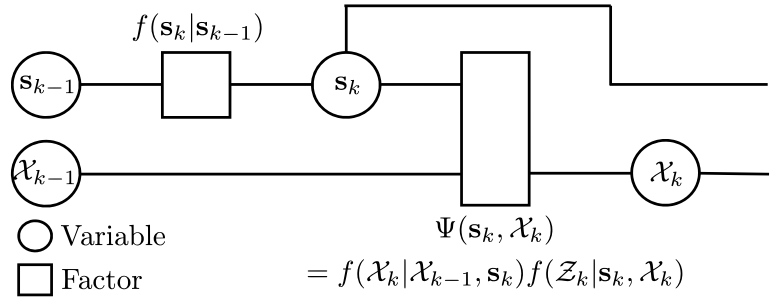

Fig. 1. Factor graph for local SLAM at time $k$ from the factorization of $f\left(\mathbf{s}_{0: k}, \mathcal{X}_{0: k} \mid \mathcal{Z}_{1: k}\right)$.

\section{A. Factorization}

The joint posterior density $f\left(\mathbf{s}_{0: K}, \mathcal{X}_{0: K} \mid \mathcal{Z}_{1: K}\right)$ is factorized as

$$
\begin{aligned}
& f\left(\mathbf{s}_{0: K}, \mathcal{X}_{0: K} \mid \mathcal{Z}_{1: K}\right) \propto \\
& f\left(\mathbf{s}_{0}\right) f\left(\mathcal{X}_{0}\right) \prod_{k=1}^{K} f\left(\mathbf{s}_{k} \mid \mathbf{s}_{k-1}\right) f\left(\mathcal{X}_{k} \mid \mathcal{X}_{k-1}, \mathbf{s}_{k}\right) f\left(\mathcal{Z}_{k} \mid \mathbf{s}_{k}, \mathcal{X}_{k}\right),
\end{aligned}
$$

where $f\left(\mathcal{Z}_{k} \mid \mathbf{s}_{k}, \mathcal{X}_{k}\right)$ is the set likelihood function. Fig 1 . shows the factor graph for the factorized joint posterior density for 1 time step. By the MP rule, $f\left(\mathcal{Z}_{k} \mid \mathbf{s}_{k}, \mathcal{X}_{k}\right)$ is integrated with $f\left(\mathcal{X}_{k} \mid \mathcal{X}_{k-1}, \mathbf{s}_{k}\right)$, and the result is denoted $\Psi\left(\mathbf{s}_{k}, \mathcal{X}_{k}\right)$.

\section{B. Prediction}

The belief $b_{k-1}\left(\mathbf{s}_{k-1}\right)$ of the variable $\mathbf{s}_{k-1}$ is sent to the factor $f\left(\mathbf{s}_{k} \mid \mathbf{s}_{k-1}\right)$. Then, a vehicle message is computed as

$$
b_{k \mid k-1}\left(\mathbf{s}_{k}\right) \propto \int b_{k-1}\left(\mathbf{s}_{k-1}\right) f\left(\mathbf{s}_{k} \mid \mathbf{s}_{k-1}\right) \mathrm{d} \mathbf{s}_{k-1},
$$

and the predicted vehicle message $b_{k \mid k-1}\left(\mathbf{s}_{k}\right)$ is sent to the variable $\mathbf{s}_{k}$. Similarly to vehicle prediction, the belief $b_{k-1}\left(\mathcal{X}_{k-1}\right)$ of the variable $\mathcal{X}_{k-1}$ is sent to the factor $\Psi\left(\mathbf{s}_{k}, \mathcal{X}_{k}\right)$. For detecting targets with the FoV in map prediction, an auxiliary message is calculated as

$$
v_{k}\left(\mathcal{X}_{k} \mid \mathbf{s}_{k}\right) \propto \int b_{k-1}\left(\mathcal{X}_{k-1}\right) f\left(\mathcal{X}_{k} \mid \mathcal{X}_{k-1}, \mathbf{s}_{k}\right) \delta \mathcal{X}_{k-1} .
$$

\section{Correction}

By product of the message $b_{k \mid k-1}\left(\mathbf{s}_{k}\right)$ of (4) and a message from the factor $\Psi\left(\mathbf{s}_{k}, \mathcal{X}_{k}\right)$, a belief of the variable $\mathbf{s}_{k}$ is updated as

$$
b_{k}\left(\mathbf{s}_{k}\right) \propto b_{k \mid k-1}\left(\mathbf{s}_{k}\right) \int v_{k}\left(\mathcal{X}_{k} \mid \mathbf{s}_{k}\right) f\left(\mathcal{Z}_{k} \mid \mathbf{s}_{k}, \mathcal{X}_{k}\right) \delta \mathcal{X}_{k} .
$$

An updated belief of the variable $\mathcal{X}_{k}$ is equal to a message from the factor $\Psi\left(\mathbf{s}_{k}, \mathcal{X}_{k}\right)$ to variable $\mathcal{X}_{k}$, which is calculated as

$$
b_{k}\left(\mathcal{X}_{k}\right) \propto \int b_{k \mid k-1}\left(\mathbf{s}_{k}\right) v_{k}\left(\mathcal{X}_{k} \mid \mathbf{s}_{k}\right) f\left(\mathcal{Z}_{k} \mid \mathbf{s}_{k}, \mathcal{X}_{k}\right) \mathrm{d} \mathbf{s}_{k} .
$$

In general, both prediction and correction lack analytical solution, due to the set integrals and the vector integrals. 


\section{LOCAL SLAM WITH CKF-PHD}

\section{A. Principle}

To avoid the intractable integrals, we make a number of approximations: integrals over random vectors are approximated using the CKF [23]; RFS densities are approximated by their PHD [12], [17], which is represented by a Gaussian mixture (GM). Standard pruning and merging, e.g., [26, Table II], is assumed to be applied to limit the number of mixture components. In particular, we will denote by $D_{k}(\mathbf{x}, m)$ a PHD for target type $m$ at time $k$. The messages for $\mathcal{X}_{k}$ are approximated by $D_{k}(\mathbf{x}, m)$ for $m=\{\mathrm{BS}, \mathrm{VA}, \mathrm{SP}\}$. We initialize $D_{0}(\mathbf{x}, \mathrm{BS})=\sum_{a=1}^{A} \delta\left(\mathbf{x}-\mathbf{x}_{\mathrm{BS}}^{(a)}\right)$, and $D_{0}(\mathbf{x}, m)=0$ for $m=\{\mathrm{VA}, \mathrm{SP}\}$. The messages for $\mathbf{s}_{k}$ are approximated by the Gaussian density, and we initialize $b_{0}\left(\mathbf{s}_{0}\right)=\mathcal{N}\left(\mathbf{s} ; \hat{\mathbf{s}}_{0}, \hat{\mathbf{U}}_{0}\right)$. We now describe the local (per vehicle) SLAM prediction and correction step. For notation convenience, we drop the vehicle index $n$.

\section{B. Prediction}

1) Vehicle (4): We decompose $\hat{\mathbf{U}}=\mathbf{C C}^{\top}$ when $b_{k-1}\left(\mathbf{s}_{k-1}\right)=\mathcal{N}\left(\mathbf{s} ; \hat{\mathbf{s}}_{k-1}, \hat{\mathbf{U}}_{k-1}\right)$ is given. Then, we generate cubature points $(\mathrm{CPs})^{4}$

$$
\mathbf{s}_{k-1}^{c}=\mathbf{C} \epsilon_{\mathbf{s}_{k}}^{c}+\hat{\mathbf{s}}_{k-1}, \quad c=1, \ldots, 2 \mathrm{~d}\left(\mathbf{s}_{k}\right),
$$

and weights $w_{k-1}^{c}=1 /\left(2 \mathrm{~d}\left(\mathbf{s}_{k}\right)\right)$. The CPs are propagated as $\mathbf{s}_{k}^{c}=\mathrm{v}\left(\mathbf{s}_{k-1}^{c}\right)$ for all $c$, and the predicted mean and covariance are calculated as

$$
\overline{\mathbf{s}}_{k}=\sum_{c=1}^{2 \mathrm{~d}\left(\mathbf{s}_{k}\right)} w_{k-1}^{c} \mathbf{s}_{k}^{c}, \quad \overline{\mathbf{U}}_{k}=\sum_{c=1}^{2 \mathrm{~d}\left(\mathbf{s}_{k}\right)} w_{k-1}^{c} \mathbf{s}_{k}^{c} \mathbf{s}_{k}^{c \top}-\overline{\mathbf{s}}_{k} \overline{\mathbf{s}}_{k}^{\top}+\mathbf{Q}_{k} .
$$

Finally, $b_{k \mid k-1}\left(\mathbf{s}_{k}\right)$ is represented as a Gaussian density: $b_{k \mid k-1}\left(\mathbf{s}_{k}\right)=\mathcal{N}\left(\mathbf{s} ; \overline{\mathbf{s}}_{k}, \overline{\mathbf{U}}_{k}\right)$.

2) Map (5): Similar to (8), we generate $\left\{\overline{\mathbf{s}}_{k}^{c}, \bar{w}_{k}^{c}\right\}_{c=1}^{2 \mathrm{~d}\left(\mathbf{s}_{k}\right)}$ from $b_{k \mid k-1}\left(\mathbf{s}_{k}\right)=\mathcal{N}\left(\mathbf{s} ; \overline{\mathbf{s}}_{k}, \overline{\mathbf{U}}_{k}\right)$. Then, the message $v_{k}\left(\mathcal{X}_{k} \mid \mathbf{s}_{k}\right)$ is also approximated by the PHD

$$
D_{k \mid k-1}\left(\mathbf{x}, m \mid \overline{\mathbf{s}}_{k}^{c}\right)=D_{k-1}(\mathbf{x}, m)+D_{k}^{b}\left(\mathbf{x}, m \mid \overline{\mathbf{s}}_{k}^{c}\right),
$$

for $m=\{\mathrm{VA}, \mathrm{SP}\}$ and each $c$, where $D_{k}^{b}\left(\mathbf{x}, m \mid \overline{\mathbf{s}}_{k}^{c}\right)$ is a birth intensity, which is also a PHD in the form of a GM. Hence, we can represent $D_{k \mid k-1}\left(\mathbf{x}, m \mid \overline{\mathbf{s}}_{k}^{c}\right)$ as

$$
D_{k \mid k-1}\left(\mathbf{x}, m \mid \overline{\mathbf{s}}_{k}^{c}\right)=\sum_{j=1}^{\bar{J}_{k}^{c}(m)} \bar{\gamma}_{k}^{c, j}(m) \mathcal{N}\left(\mathbf{x} ; \overline{\mathbf{x}}_{k}^{c, j}(m), \overline{\mathbf{P}}_{k}^{c, j}(m)\right) .
$$

\section{Correction}

1) Vehicle (6): We first compute the weighted sum of the predicted map PHDs for each $\overline{\mathbf{s}}_{k}^{c}$ (for $m=\left\{\mathrm{VA}^{(a)}, \mathrm{SP}\right\}$ ):

$$
D_{k \mid k-1}(\mathbf{x}, m)=\sum_{c=1}^{2 \mathrm{~d}\left(\mathbf{s}_{k}\right)} \bar{w}_{k}^{c} D_{k \mid k-1}\left(\mathbf{x}, m \mid \overline{\mathbf{s}}_{k}^{c}\right),
$$

\footnotetext{
${ }^{4}$ We define $\mathrm{d}(\star)$ as the number of unknown variables of $\star$, and $\epsilon_{\star}^{c}$ is the $c$ th column vector of the matrix $\sqrt{\mathrm{d}(\star)}\left[\mathbf{I}^{\mathrm{d}(\star)},-\mathbf{I}^{\mathrm{d}(\star)}\right] \in \mathbb{R}^{\mathrm{d}(\star) \times 2 \mathrm{~d}(\star)}$, where $\mathbf{I}^{\mathrm{d}(\star)} \in \mathbb{R}^{\mathrm{d}(\star) \times \mathrm{d}(\star)}$ is the identity matrix.

${ }^{5}$ For birth generation, the Gaussian density is calculated for all $\mathbf{z}_{k, l} \in \mathcal{Z}_{k}$, and the visible birth is selected. For birth weight, we adopt an adaptive birth density [27]. Details on both are found in [6, Sec. III].
}

which in this work is represented as a GM:

$$
D_{k \mid k-1}(\mathbf{x}, m)=\sum_{j=1}^{\bar{J}_{k}(m)} \bar{\gamma}_{k}^{j}(m) \mathcal{N}\left(\mathbf{x} ; \overline{\mathbf{x}}_{k}^{j}(m), \overline{\mathbf{P}}_{k}^{j}(m)\right) .
$$

Note that, since the base station is known, $D_{k \mid k-1}(\mathbf{x}, \mathrm{BS})=$ $D_{k-1}(\mathbf{x}, \mathrm{BS})$. Instead of using a particle filter implementation of the vehicle correction, we apply a low-complexity CKF [23], whereby the joint posterior of the vehicle and detected targets are estimated, and targets are marginalized out. This requires knowledge of the data association, which is described in Sec. IV-D. Assuming the data association returns $J_{k}^{\mathrm{M}}$ targets that were associated to measurements, we introduce the random vector $\mathbf{g}_{k}=\left[\mathbf{s}_{k}^{\top}, \ldots,\left(\mathbf{x}_{k}^{J_{k}^{\mathrm{M}}}\right)^{\top}\right]^{\top}$, which has an a priori Gaussian distribution with mean $\overline{\mathbf{g}}_{k}=\left[\overline{\mathbf{s}}_{k}^{\top}, \ldots,\left(\overline{\mathbf{x}}_{k}^{J_{k}^{\mathrm{M}}}\right)^{\top}\right]^{\top}$ and covariance matrix $\overline{\mathbf{G}}_{k}=\operatorname{blkdiag}\left(\overline{\mathbf{U}}_{k}, \ldots, \overline{\mathbf{P}}_{k}^{J_{k}^{\mathrm{M}}}\right)$. Given the measurements $\tilde{\mathbf{z}}_{k}=\left[\mathbf{z}_{k, l(1)}^{\top}, \ldots, \mathbf{z}_{k, l\left(J_{k}^{\mathrm{M}}\right)}^{\top}\right]^{\top}$, where $l(j)$ is the measurement index $l$ corresponding to the matched target $j$, the posterior of $\mathbf{g}_{k}$ given $\tilde{\mathbf{z}}_{k}$ can be obtained from the CKF. Similar to (8), we generate cubature points: $\left\{\mathbf{g}_{k}^{g}\right\}_{g=1}^{2\left(\overline{\mathbf{g}}_{k}\right)}$, where $\mathbf{g}_{k}^{g}=\left[\left(\mathbf{s}_{k}^{g}\right)^{\top}, \ldots,\left(\mathbf{g}_{k}^{g, J_{k}^{\mathrm{M}}}\right)^{\top}\right]^{\top}$. We denote $m(j)$ the type of matched target $j$. We compute the Kalman gain $\mathbf{K}_{k}=\mathbf{P}_{\mathbf{g z}, k} \mathbf{P}_{\mathbf{z z}, k}^{-1}$, where the cross-covariance matrix $\mathbf{P}_{\mathbf{g z}, k}$ and the predicted covariance matrix $\mathbf{P}_{\mathbf{z z}, k}$ are respectively calculated as

$$
\begin{aligned}
& \mathbf{P}_{\mathbf{g z}, k}=\frac{1}{2 \mathrm{~d}\left(\overline{\mathbf{g}}_{k}\right)} \sum_{g=1}^{2 \mathrm{~d}\left(\overline{\mathbf{g}}_{k}\right)} \mathbf{g}_{k}^{g}\left(\overline{\mathbf{z}}_{k}^{g}\right)^{\top}-\overline{\mathbf{g}}_{\mathrm{M}, k} \overline{\mathbf{z}}_{\mathrm{M}, k}^{\top}, \\
& \mathbf{P}_{\mathbf{z z}, k}=\frac{1}{2 \mathrm{~d}\left(\overline{\mathbf{g}}_{k}\right)} \sum_{g=1}^{2 \mathrm{~d}\left(\overline{\mathbf{g}}_{k}\right)} \overline{\mathbf{z}}_{k}^{g}\left(\overline{\mathbf{z}}_{k}^{g}\right)^{\top}-\overline{\mathbf{z}}_{\mathrm{M}, k} \overline{\mathbf{z}}_{\mathbf{M}, k}^{\top}+\mathbf{R}_{k},
\end{aligned}
$$

where $\overline{\mathbf{z}}_{k}^{g}=\left[\mathrm{h}\left(\mathbf{s}_{k}^{g}, \mathbf{g}_{k}^{g, 1}, m(1)\right)^{\top}, \ldots, \mathrm{h}\left(\mathbf{s}_{k}^{g}, \mathbf{g}_{k}^{g, J_{k}^{\mathrm{M}}}, m\left(J_{k}^{\mathrm{M}}\right)\right)^{\top}\right]^{\top}$, $\overline{\mathbf{g}}_{\mathrm{M}, k}=\sum_{g=1}^{2 \mathrm{~d}\left(\overline{\mathbf{g}}_{k}\right)} \mathbf{g}_{k}^{g} /\left(2 \mathrm{~d}\left(\overline{\mathbf{g}}_{k}\right)\right)$, and $\overline{\mathbf{z}}_{\mathrm{M}, k}=\sum_{g=1}^{2 \mathrm{~d}\left(\overline{\mathbf{g}}_{k}\right)} \overline{\mathbf{z}}_{k}^{g} /\left(2 \mathrm{~d}\left(\overline{\mathbf{g}}_{k}\right)\right)$.

The posterior mean $\hat{\mathbf{g}}_{k}$ and covariance $\hat{\mathbf{G}}_{k}$ are computed as

$$
\begin{gathered}
\hat{\mathbf{g}}_{k}=\overline{\mathbf{g}}_{k}+\mathbf{K}_{k}\left(\overline{\mathbf{z}}_{\mathrm{M}, k}-\tilde{\mathbf{z}}_{k}\right), \\
\hat{\mathbf{G}}_{k}=\overline{\mathbf{G}}_{k}-\mathbf{K}_{k} \mathbf{P}_{\mathbf{z z}, k} \mathbf{K}_{k}^{\top} .
\end{gathered}
$$

Finally, from the estimated joint posterior, $b_{k}\left(\mathbf{s}_{k}\right)=$ $\mathcal{N}\left(\mathbf{s} ; \hat{\mathbf{s}}_{k}, \hat{\mathbf{U}}_{k}\right)$ is extracted.

2) Map (7): The map PHD is updated for each $c$ and $m$ [17]:

$$
\begin{aligned}
D_{k}\left(\mathbf{x}, m \mid \overline{\mathbf{s}}_{k}^{c}\right) & =D_{k \mid k-1}\left(\mathbf{x}, m \mid \overline{\mathbf{s}}_{k}^{c}\right)\left(1-p_{\mathrm{D}}\left(\mathbf{x}, m, \overline{\mathbf{s}}_{k}^{c}\right)\right) \\
& +\sum_{\mathbf{z} \in \mathcal{Z}_{k}} \frac{\nu^{c}\left(\mathbf{z}, \mathbf{x}, m, \overline{\mathbf{s}}_{k}^{c}\right)}{c(\mathbf{z})+\sum_{m^{\prime}} \int \nu^{c}\left(\mathbf{z}, \mathbf{x}^{\prime}, m^{\prime}, \overline{\mathbf{s}}_{k}^{c}\right) \mathrm{d} \mathbf{x}^{\prime}},
\end{aligned}
$$

where $\nu\left(\mathbf{z}, \mathbf{x}, m, \overline{\mathbf{s}}_{k}^{c}\right)=\sum_{j=1}^{\bar{J}_{k}^{c}(m)} \nu^{j}\left(\mathbf{z}, \mathbf{x}, m, \overline{\mathbf{s}}_{k}^{c}\right)$. We denote by $b(\mathbf{z})$ the index of the birth component corresponding to $\mathbf{z}$. Here, if $b(\mathbf{z})=j$,

$$
\nu^{j}\left(\mathbf{z}, \mathbf{x}, m, \overline{\mathbf{s}}_{k}^{c}\right)=\bar{\gamma}_{k}^{c, j}(m) \mathcal{N}\left(\mathbf{x} ; \overline{\mathbf{x}}_{k}^{c, j}(m), \overline{\mathbf{P}}_{k}^{c, j}(m)\right),
$$


otherwise,

$$
\begin{aligned}
& \nu^{j}\left(\mathbf{z}, \mathbf{x}, m, \overline{\mathbf{s}}_{k}^{c}\right)=p_{\mathrm{D}, k}^{c, j}(m) \bar{\gamma}_{k}^{c, j}(m) \mathcal{N}\left(\mathbf{x} ; \hat{\mathbf{x}}_{k}^{c, j}(m), \hat{\mathbf{P}}_{k}^{c, j}(m)\right) \\
& \times \mathcal{N}\left(\mathbf{z} ; \mathbf{h}\left(\overline{\mathbf{s}}_{k}^{c}, \overline{\mathbf{x}}_{k}^{c, j}(m), m\right), \mathbf{S}_{\mathbf{z z}, k}^{j}(m)\right) .
\end{aligned}
$$

where $p_{\mathrm{D}, k}^{c, j}(m)$ is an adaptive detection probability. We use the adaptive $p_{\mathrm{D}, k}^{c, j}(m)$ proposed in [6], which avoids problems with misdetected targets near the edge of the sensor's FoV; for details refer to [6].

The updated Gaussian density $\mathcal{N}\left(\mathbf{x} ; \hat{\mathbf{x}}_{k, j}^{c, j}(m), \hat{\mathbf{P}}_{k}^{c, j}(m)\right)$ and the predicted likelihood $\mathcal{N}\left(\mathbf{z} ; \mathrm{h}\left(\overline{\mathbf{s}}_{k}^{c}, \overline{\mathbf{x}}_{k}^{c, j}(m), m\right), \mathbf{S}_{\mathbf{z z}, k}^{j}(m)\right)$ are calculated by the CKF [23]. Similar to (10), then, $b_{k}\left(\mathcal{X}_{k}\right)$ is represented by calculating the average map PHD $D_{k}(\mathbf{x}, m)=$ $\sum_{c=1}^{2 \mathrm{~d}\left(\mathbf{s}_{k}\right)} \bar{w}_{k}^{c} D_{k}\left(\mathbf{x}, m \mid \overline{\mathbf{s}}_{k}^{c}\right)$, where $\bar{w}_{k}^{c}=1 /\left(2 \mathrm{~d}\left(\mathbf{s}_{k}\right)\right)$.

\section{Data Association}

The vehicle correction step relies on a data association between targets and measurements. We select targets from the PHD that satisfy $\bar{\gamma}_{k}^{j}(m) \geq T_{m}$. We denote by $J^{\mathrm{S}}(m)$ the number of selected targets, and denote by $m^{j}$ the type of the selected component.

Next, under the assumption that the targets are sufficiently sparse, we try to match the selected targets to the measurements using ellipsoidal gating [28]. The ellipsoidal gating distance is defined as

$$
\begin{aligned}
\left(d_{k, l}^{j}\right)^{2} & =\left(\mathbf{z}_{k, l}-\mathrm{h}\left(\overline{\mathbf{s}}_{k}^{e, j}, \overline{\mathbf{x}}_{k}^{e, j}, m^{j}\right)\right)^{\top}\left(\mathbf{S}_{k}^{j}\right)^{-1} \\
& \times\left(\mathbf{z}_{k, l}-\mathrm{h}\left(\overline{\mathbf{s}}_{k}^{e, j}, \overline{\mathbf{x}}_{k}^{e, j}, m^{j}\right)\right),
\end{aligned}
$$

where $\overline{\mathbf{s}}_{k}^{e, j}$ and $\overline{\mathbf{x}}_{k}^{e, j}(m)$ are generated (similar to (8)) from $\mathcal{N}\left(\mathbf{e}, \mathbf{e}_{k}^{j}, \mathbf{E}_{k}^{j}\right)$. Here, $\mathbf{e}_{k}^{j}=\left[\overline{\mathbf{s}}_{k}^{\top},\left(\overline{\mathbf{x}}_{k}^{j}\right)^{\top}\right]^{\top}$ and $\mathbf{E}_{k}^{j}=$ blkdiag $\left(\overline{\mathbf{U}}_{k}, \overline{\mathbf{P}}_{k}^{j}\right)$. The innovation covariance $\mathbf{S}_{k}^{j}$ is calculated by the standard CKF [23]:

$$
\mathbf{S}_{k}^{j}=\frac{1}{2 \mathrm{~d}\left(\mathbf{e}_{k}^{j}\right)} \sum_{e=1}^{2 \mathrm{~d}\left(\mathbf{e}_{k}^{j}\right)} \overline{\mathbf{z}}_{k}^{e, j}\left(\overline{\mathbf{z}}_{k}^{e, j}\right)^{\top}-\overline{\mathbf{z}}_{\mathrm{S}, k}^{j}\left(\overline{\mathbf{z}}_{\mathrm{S}, k}^{j}\right)^{\top}+\mathbf{R}_{k},
$$

where

$$
\overline{\mathbf{z}}_{k}^{e, j}=\mathrm{h}\left(\overline{\mathbf{s}}_{k}^{e}, \overline{\mathbf{x}}_{k}^{e, j}, m^{j}\right) \text {, and } \overline{\mathbf{z}}_{\mathrm{S}, k}^{j}=1 /\left(2 \mathrm{~d}\left(\mathbf{e}_{k}^{j}\right)\right) \sum_{e=1}^{2 \mathrm{~d}\left(\mathbf{e}_{k}^{j}\right)} \overline{\mathbf{z}}_{k}^{e, j} .
$$

For target $j$, we consider all measurements inside the gate, $\left(d_{k, l}^{j}\right)^{2}<T_{\mathrm{G}}$, where $T_{\mathrm{G}}$ is a gating threshold, computed by using the Chi-square CDF with gate probability $p_{\mathrm{G}}$ and degrees of freedom $\operatorname{dim}(\mathbf{z})$. Finally, among measurements $\mathbf{z}_{k, l} \in \mathcal{Z}_{k}$, we associate to target $j$ the closest measurement $l^{\star}$, where $l^{\star}=\operatorname{argmin}_{l}\left\{\left(d_{k, l}^{j}\right)^{2}\right\}<T_{\mathrm{G}}$.

\section{MAP Fusion fOR MUlti-CEll SLAM}

The method presented in Section IV allows local SLAM per vehicle. In order to deal with the multi-cell multi-vehicle scenario without performing global SLAM, we must define how maps are managed, fused, and shared. Note that VA map information is managed separately for each BS since the VA is BS-specific in the map (but the reflecting surface is not specific in the map), while SP map information can be managed jointly. We denote by $D_{k}^{\mathrm{CC}}(\mathbf{x}, m)$ a PHD of the global map at the control center. We initialize $D_{k}^{\mathrm{CC}}(\mathbf{x}, m)=0$, for $m \in\left\{\mathrm{VA}^{(1)}, \ldots, \mathrm{VA}^{(A)}, \mathrm{SP}\right\}$.

\section{A. Uplink Transmission}

Vehicle $n$ sends following map information to the linked BS: i) average map PHD of (16); and ii) the accumulated FoV from at time $k^{*}$ (last uplink transmission for map fusion). The accumulated FoV is represented as

$\mathcal{F}_{k}^{(n)}(m)=\left\{\mathbf{x}: \exists k^{\prime} \in\left(k^{*}, k\right], \max _{c}\left\{p_{\mathrm{D}, k^{\prime}}\left(\mathbf{x}, m, \hat{\mathbf{s}}_{k^{\prime}}^{c}\right)\right\} \geq \gamma_{\mathbf{D}}\right\}$,

where $\gamma_{\mathbf{D}}$ is a detection threshold $\left(\gamma_{\mathbf{D}}\right.$ closed to 1$)$. The BS relays the $D_{k}^{(n)}(\mathbf{x}, m)$ and $\mathcal{F}_{k}^{(n)}(m)$ to the CC.

\section{B. Map Fusion for Global Map}

Let $D_{k \mid k-1}^{\mathrm{CC}}(\mathbf{x}, m)=D_{k-1}^{\mathrm{CC}}(\mathbf{x}, m)$ denote the predicted global map PHD. To handle target misdetections well, we use the AA method [21], [22] for map fusion. The CC updates the global map PHD by fusing the predicted global map PHD $D_{k \mid k-1}^{\mathrm{CC}}(\mathbf{x}, m)$ with the received local map PHD $D_{k}^{(n)}(\mathbf{x}, m)$ :

$$
D_{k}^{\mathrm{CC}}(\mathbf{x}, m)=\beta_{p} D_{k \mid k-1}^{\mathrm{CC}}(\mathbf{x}, m)+\beta_{a} D_{k}^{(n)}(\mathbf{x}, m),
$$

where $\beta_{p} \geq 0$ and $\beta_{a} \geq 0$ are adaptive weights for the two PHDs, representing the relative importance between them, with $\beta_{p}+\beta_{a}=1$. For newly detected targets, the weight in the PHD is reduced by $\beta_{a}$ because doing so improves the handling of false alarms. We determine $\beta_{p}$ and $\beta_{a}$ separately for each GM component by a matching algorithm, as in [6, Sec. IV-B].

\section{Downlink Transmission}

The CC sends the updated global map PHD $D_{k}^{\mathrm{CC}}(\mathbf{x}, m)$ of (22) back to all BSs and each BS transmits it to the vehicle. Then, vehicle $n$ overwrites $D_{k}^{\mathrm{CC}}(\mathbf{x}, m)$ to the updated local map PHD as

$$
D_{k}^{(n)}(\mathbf{x}, m)=D_{k}^{\mathrm{CC}}(\mathbf{x}, m) .
$$

Maps for other vehicles are unchanged.

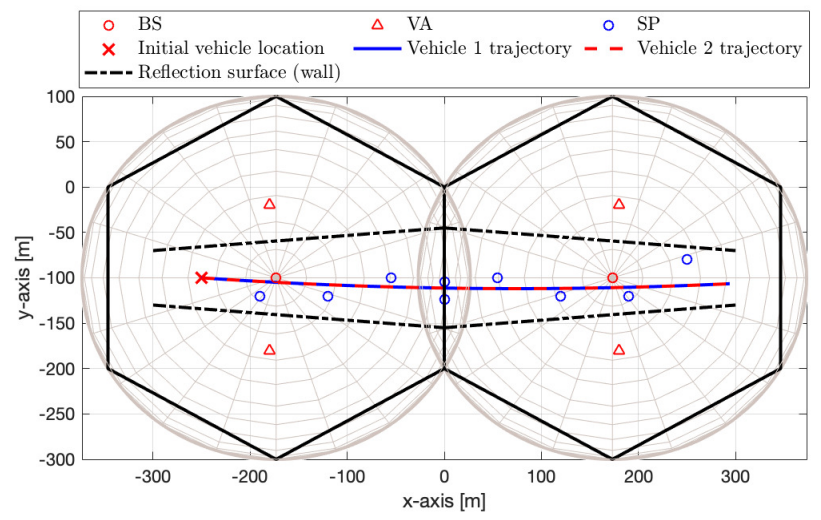

Fig. 2. Vehicle trajectories and map of environment consisting of two small cells. 


\section{NUMERICAL RESUlTS}

\section{A. Simulation Setup}

We deploy two small cells (see, Fig. 2), and we set $r_{\mathrm{SC}}=200 \mathrm{~m}$. Two BSs are located at $[-173.2,-100,40]^{\top}$ and $[173.2,-100,40]^{\top}$, with unit $\mathrm{m}$, and we set $r_{\max }=220 \mathrm{~m}$. For BS 1 , two VAs are located at $[-179.92,-19.43,40]^{\top}$ and $[-179.92,-180.57,40]^{\top}$, and for BS 2 , two VAs are located at $[179.92,-19.43,40]^{\top}$ and $[179.92,-180.57,40]^{\top}$. Nine SPs are deployed near the road, and vertical location of the SP is generated uniformly between 0 and $40 \mathrm{~m}$.

We consider the following scenario for vehicle movement and map fusion. We adopt the mobility model [24, Chap. 5] for considering the vehicle movement, and set $\mathbf{q}_{k}=[0.5,0.5,0,0.01,0,0,0.5]^{\top}$, with units $\mathrm{m}, \mathrm{m}, \mathrm{m}$, $\mathrm{rad}, \mathrm{m} / \mathrm{s}, \mathrm{rad} / \mathrm{s}$, and $\mathrm{m}$. The time interval $\Delta$ is set to $0.5 \mathrm{~s}$. The initial state of vehicle 1 is set to $\mathbf{s}_{0}^{(1)}=$ $[-250,-100,0 ; 6.21,22.22,0.005,300]^{\top}$, with units as the process noise. During 50 time steps, vehicle 1 moves to left to right as shown in Fig. 2. For vehicle 1, the proposed map fusion routine except for downlink transmission is performed. After vehicle 1 leaves the small cells, vehicle 2 appears at the same initial location. Vehicle 2 has movement same as vehicle 1 , but vehicle 2 performs the full map fusion routine. The standard deviation of initial prior $f\left(\mathbf{s}_{0}\right)$ for all vehicles is set to $[3,3,0 ; 0.052,0,0,3]^{\top}$, with units as the process noise.

We set $\mathbf{r}_{k}=[0.1,0.01,0.01,0.01,0.01]^{\top}$, with units $\mathrm{m}$, $\mathrm{rad}$, rad, rad, and rad. The target is detected by the vehicle within the target $\mathrm{FoV}^{6}$, modeled as the distance range. For the FoV, we set $r_{\mathrm{BS}}=r_{\mathrm{VA}}=220 \mathrm{~m}$ and $r_{\mathrm{SP}}=50$ $\mathrm{m}$. The detection probability $p_{\mathrm{D}}$ is set to 0.9 . The average of the number of clutter measurements follows the Poisson distribution with $\lambda=1$, and the clutter intensity is modeled as $c(\mathbf{z})=\lambda /\left(4 r_{\max } \pi^{4}\right)$ (about $\left.1.17 \times 10^{-5}\right)$. To make our PHD filter robust to false alarms, the birth intensity is also set to about $1.17 \times 10^{-5}$ same as the clutter intensity. For $\mathrm{DA}$, the gating probability for ellipsoidal gating $p_{\mathrm{G}}$ is set to 0.99 . Parameters for the pruning and merging are summarized as follows: truncation threshold $T=10^{-4}$, merging threshold $U=49$, and maximum allowable Gaussians $J_{\max }=50$.

For performance evaluation, the target of which weight is larger than the target detection threshold is chosen, and we set $T_{\mathrm{VA}}=0.8$ and $T_{\mathrm{SP}}=0.6$. We utilize the root mean square error (RMSE) for measuring the accuracy of vehicle state estimation, and the average of the generalized optimal subpattern assignment (GOSPA) distance [29] for the measuring mapping accuracy. For GOSPA distance calculation, we set the cut off distance $q_{c}=20$, power parameter $q_{p}=2$, and $q_{a}=2$. The results in Sec. VI-B are obtained by averaging over 10 Monte Carlo simulations.

\section{B. Discussions}

1) Vehicle: To demonstrate the contributions of this paper, we evaluated vehicle state estimation for two cases as follows:

\footnotetext{
${ }^{6}$ We denote $r_{\mathrm{BS}}$ the BS FoV, $r_{\mathrm{VA}}$ the VA FoV, $r_{\mathrm{SP}}$ the SP FoV. Note that $r_{\mathrm{BS}}=r_{\mathrm{VA}}=r_{\mathrm{SC}}$ by geometric relations.
}

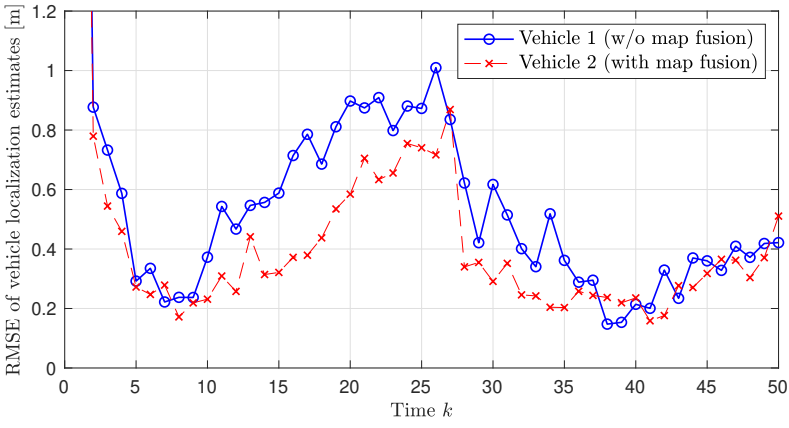

Fig. 3. RMSE of vehicle location estimates over time steps.

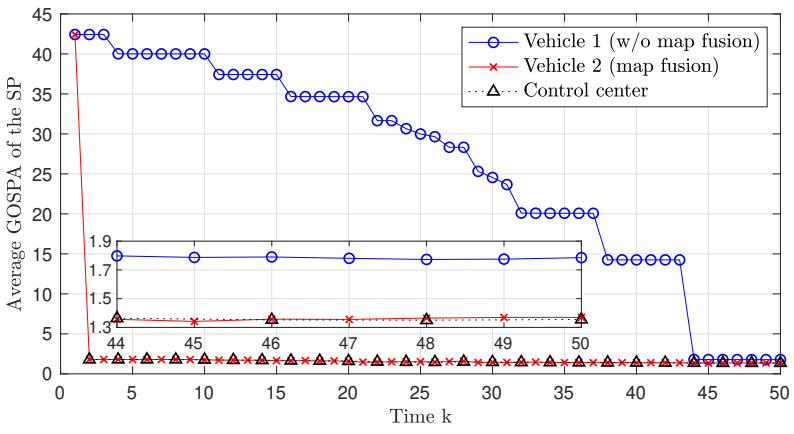

(a)

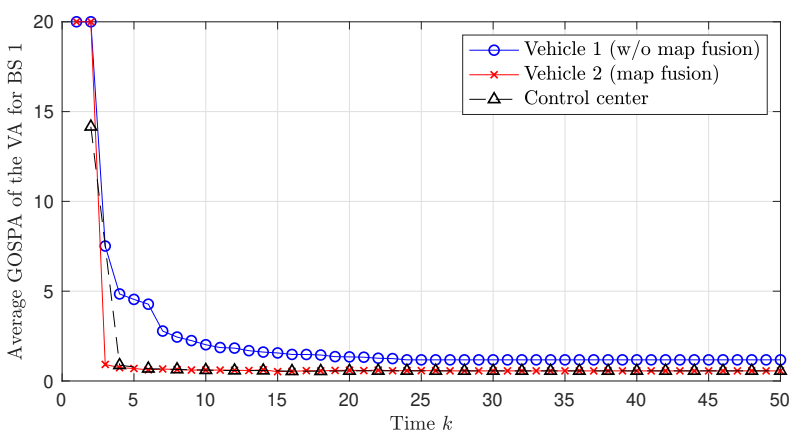

(b)

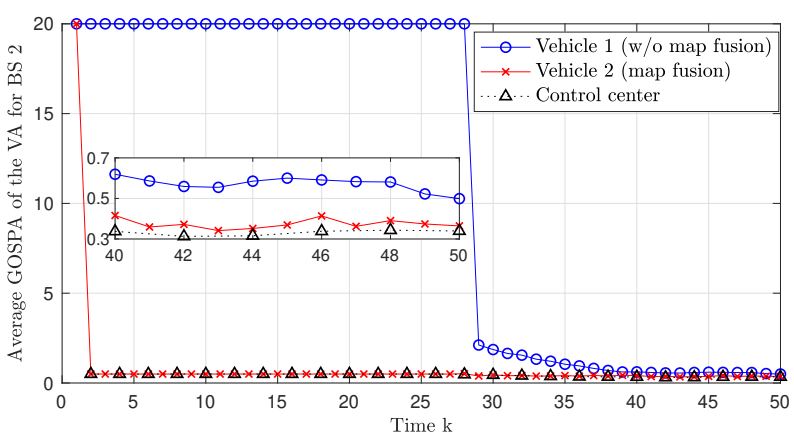

(c)

Fig. 4. Average GOSPA of the (a) SP (b) VA in small cell 1 (BS 1) (c) VA for in small cell 2 (BS 2).

case i) the vehicle only performs local SLAM (Sec. IV); and case ii) the vehicle performs the proposed 5G multi-cell SLAM (which, i.e., indicates vehicle 2 in Sec. VI-A). Fig. 3 shows the the accuracy of vehicle location estimates over time steps, 
which is evaluated by the RMSE. Note that for vehicle 2 , the time axis is $k-50$. In case ii), there is performance gain compared to case i). It is clearly shown that vehicle 2 , entered the environment after $k=50$, has benefit from the map built by the vehicle 1 . The RMSE increase between $k=10$ and $k=30$ is due to the geometrical configuration of SPs. The RMSEs of the heading and clock bias estimates show similar gains (results now shown).

2) Map: Fig. 4 shows the average GOSPA for case i) vehicle 1 , case ii) vehicle 2 , and the global map in the CC. In Fig. 4a, the average GOSPA of the SP is shown. When there is no available map information (for vehicle 1), the GOSPA goes down as the vehicle moves in the environment. For vehicle 2, which can exploit the the global map built by vehicle 1, the GOSPA reduces faster and can further improve mapping accuracy compared to vehicle 1 . In Fig. $4 \mathrm{~b}$ and $4 \mathrm{c}$, the average GOSPAs of the VA in small cell 1 and 2 are shown, respectively. As the SP GOSPA, for vehicle 1, the VA GOSPAs go down as the vehicle moves in the environment. For vehicle 2, the GOSPA also reduces faster and also has the mapping gain compared to vehicle 1 . There are slight or no performance gains for the global map, however the mapping accuracy is maintained when the linked BS to the vehicle changes, which is the one of aforementioned challenges of the multi-cell scenario.

\section{CONCLUSIONS}

We proposed a novel joint CKF-PHD filter and map fusion routine for $5 \mathrm{G}$ multi-cell SLAM. The method combines principles from random finite set theory and message passing on factor graphs, and was demonstrated in a 2-cell scenario. Performance gains in both vehicle localization accuracy and mapping accuracy were observed from inter-vehicle cooperation through map sharing with a control center. While the proposed filter does not capture dependencies between vehicle trajectory and the map, it has far lower complexity than a full RBPF.

\section{ACKNOWLEDGMENTS}

This work was supported, in part, by the MSIT (Ministry of Science and ICT), Korea, under the ITRC (Information Technology Research Center) support program (IITP-2019-2017-0-01637) supervised by the IITP (Institute for Information \& Communications Technology Planning \& Evaluation), by the Samsung Research Funding and Incubation Center of Samsung Electronics under Project Number SRFC-IT-1601-09, and by the Swedish Research Council under grant No. 2018-03701.

\section{REFERENCES}

[1] H. Wymeersch, G. Seco-Granados, G. Destino, D. Dardari, and F. Tufvesson, " $5 \mathrm{G}$ mm-Wave positioning for vehicular networks," IEEE Wireless Commun., vol. 24, no. 6, pp. 80-86, Dec. 2018.

[2] H. Wymeersch, N. Garcia, H. Kim, G. Seco-Granados, S. Kim, F. Wen, and M. Fröhle, "5G mmWave downlink vehicular positioning," in Proc. IEEE Global Commun. Conf. (GLOBECOM), Abu Dhabi, UAE, Dec. 2018, pp. 206-212.

[3] R. Mendrzik, H. Wymeersch, and G. Bauch, "Joint localization and mapping through millimeter wave MIMO in $5 \mathrm{G}$ systems-extended version," arXiv preprint arXiv:1804.04417, 2018.

[4] R. Mendrzik, H. Wymeersch, G. Bauch, and Z. Abu-Shaban, "Harnessing NLOS components for position and orientation estimation in 5G millimeter wave MIMO," IEEE Trans. Wireless Commun., vol. 18, no. 1, pp. 93-107, 2019.
[5] H. Kim, H. Wymeersch, N. Garcia, G. Seco-Granados, and S. Kim, "5G mmWave vehicular tracking," in Proc. IEEE 52nd Asilomar Conf. Signals, Syst., Comput., Pacific Grove, CA, USA, Oct. 2018, pp. 541547.

[6] H. Kim, K. Granström, L. Gao, G. Battistelli, S. Kim, and H. Wymeersch, "5G mmWave cooperative positioning and mapping using multimodel PHD," arXiv preprint arXiv:1907.09806, 2019.

[7] A. Yassin, Y. Nasser, A. Y. Al-Dubai, and M. Awad, "MOSAIC: Simultaneous localization and environment mapping using mmWave without a-priori knowledge," IEEE Access, vol. 6, pp. 68 932-68 947, Nov. 2018.

[8] M. Aladsani, A. Alkhateeb, and G. C. Trichopoulos, "Leveraging mmWave imaging and communications for simultaneous localization and mapping," in Proc. 2019 IEEE Int. Conf. Acoust., Speech Signal Process. (ICASSP), Brighton, UK, May 2019, pp. 4539-4543.

[9] H.-A. Loeliger, "An introduction to factor graphs," IEEE Signal Process. Mag., vol. 21, no. 1, pp. 28-41, Jan. 2004.

[10] F. R. Kschischang, B. J. Frey, and H.-A. Loeliger, "Factor graphs and the sum-product algorithm," IEEE Trans. Inf. Theory, vol. 47, no. 2, pp. 498-519, Feb. 2001.

[11] J. Mullane, B.-N. Vo, M. D. Adams, and B.-T. Vo, "A random-finite-set approach to Bayesian SLAM," IEEE Trans. Robot., vol. 27, no. 2, pp. 268-282, Apr. 2011.

[12] R. Mahler, Statistical multisource-multitarget information fusion. Norwood, MA, USA: Artech House, 2007.

[13] M. Fatemi, K. Granström, L. Svensson, F. Ruiz, and L. Hammarstrand, "Poisson multi-Bernoulli mapping using Gibbs sampling," IEEE Trans. Signal Process., vol. 65, no. 11, pp. 2814-2827, Jun. 2017.

[14] G. Battistelli, L. Chisci, and A. Laurenzi, "Random set approach to distributed multivehicle SLAM," IFAC-PapersOnLine, vol. 50, no. 1 , pp. 2457 - 2464, 2017, 20th IFAC World Congress.

[15] S. Pasha, B.-N. Vo, H. Tuan, and W.-K. Ma, "A Gaussian mixture PHD filter for jump Markov system models," IEEE Trans. Aerosp. Electron. Syst., vol. 45, no. 3, pp. 919-936, Jul. 2009.

[16] R. Mahler, "On multitarget jump-Markov filters," in Proc. 15th Int. Conf. Inf. Fusion (FUSION), Singapore, Jul. 2012, pp. 149-156.

[17] _ , "Multitarget Bayes filtering via first-order multi target moments," IEEE Trans. Aerosp. Electron. Syst., vol. 39, no. 4, pp. 1152-1178, Oct. 2003.

[18] S. Li, G. Battistelli, L. Chisci, W. Yi, B. Wang, and L. Kong, "Multisensor multi-object tracking with different fields-of-view using the $1 \mathrm{mb}$ filter," in Proc. 21st Int. Conf. Inf. Fusion (FUSION), Cambridge, U.K., Jul. 2018, pp. 1201-1208.

[19] G. Li, G. Battistelli, W. Yi, and L. Kong, "Distributed multi-sensor multi-view fusion based on generalized covariance intersection," Signal Process., vol. 166, p. 107246, 2020.

[20] R. P. Mahler, "Optimal/robust distributed data fusion: A unified approach," in Proc. SPIE Defense and Security Symp., 2000, pp. 128-139.

[21] T. Li, V. Elvira, H. Fan, and J. M. Corchado, "Local-diffusion-based distributed SMC-PHD filtering using sensors with limited sensing range," IEEE Sensors J., vol. 19, no. 4, pp. 1580-1589, 2018.

[22] L. Gao, G. Battistelli, and L. Chisci, "Multiobject fusion with minimum information loss," arXiv preprint arXiv:1903.04239, 2019.

[23] I. Arasaratnam and S. Haykin, "Cubature Kalman filters," IEEE Trans. Autom. Control, vol. 54, no. 6, pp. 1254-1269, Jun. 2009.

[24] S. Thrun, W. Burgard, and D. Fox, Probabilistic Robotics (Intelligent Robotics and Autonomous Agents Series). MIT Press, 2005.

[25] R. W. Heath, N. González-Prelcic, S. Rangan, W. Roh, and A. M. Sayeed, "An overview of signal processing techniques for millimeter wave MIMO systems," IEEE J. Sel. Topics Signal Process., vol. 10, no. 3, pp. 436-453, Apr. 2016.

[26] B.-N. Vo and W.-K. Ma, "The Gaussian mixture probability hypothesis density filter," IEEE Trans. Signal Process., vol. 54, no. 11, pp. 40914104, Nov. 2006.

[27] B. Ristic, D. Clark, B. N. Vo, and B. T. Vo, "Adaptive target birth intensity for PHD and CPHD filters," IEEE Transactions on Aerospace and Electronic Systems, vol. 48, no. 2, pp. 1656-1668, 2012.

[28] K. Panta, B.-N. Vo, and S. Singh, "Novel data association schemes for the probability hypothesis density filter," IEEE Trans. Aerosp. Electron. Syst., vol. 43, no. 2, pp. 556-570, 2007.

[29] A. S. Rahmathullah, A. F. García Fernández, and L. Svensson, "Generalized optimal sub-pattern assignment metric," in Proc. 20th Int. Conf. Inf. Fusion (FUSION), Xian, China, Jul. 2017, pp. 1-8. 